\title{
Architettura del Settecento a Episcopio di Sarno. La scala aperta nella residenza tardobarocca tra capitale e provincia
}

\author{
Rosa M. Giusto \\ Università degli Studi di Firenze \\ rmgiusto@libero.it
}

\begin{abstract}
La scala aperta nella residenza tardobarocca in Campania rappresenta uno dei temi che hanno maggiormente inciso sulla progettazione degli spazi privati tra Seicento e Settecento. II saggio indaga le relazioni intercorrenti tra il sistema delle scale aperte adottate in ambiente napoletano ed esempi significativi realizzati a Episcopio di Sarno (Sa) influenzati da un analogo processo di 'metamorfosi' della residenza privata, oltre che dall'attività di maestri quali Ferdinando Sanfelice, Domenico Antonio Vaccaro, Angelo e Francesco Solimene. II quadro che si delinea è di grande interesse per una più estesa indagine conoscitiva del territorio campano dove emergono edifici tardobarocchi inediti nei quali il sistema delle scale aperte e le sue connessioni col cortile e col giardino retrostante sono impiegati come elementi di articolazione spaziale e distributiva degli ambienti, come spazi funzionali destinati agli usi padronali del palazzo, oltre che per sottolineare il prestigio delle famiglie committenti interessate a promuovere il proprio nome ben oltre i più angusti confini locali.
\end{abstract}

PAROLE CHIAVE: Scala aperta, Episcopio di Sarno, Residenza, Tardobarocco, Ferdinando Sanfelice.

\section{Architecture of the Eighteenth Century in Episcopio di Sarno. The open Staircase in the Late-Baroque Residences in the Capital and the Province}

ABSTRACT: The use of the open staircase in the baroque and late-baroque residence in Campania is one of the topics that have most influenced the design of private spaces between the Seventeenth and Eighteenth centuries. The paper points out the relationships between the system of open staircases adopted in Naples and significant examples built in Episcopio di Sarno (Sa) and influenced by a similar process of 'metamorphosis' of the private residence, as well as by the activity of masters like Ferdinando Sanfelice, Domenico Antonio Vaccaro, Angelo and Francesco Solimene. The outlined picture is of great interest for a more extensive cognitive investigation of the architecture in Campania, where it is possible to observe unpublished late Baroque buildings in which the system of the open staircases and its connections with the courtyard and the rear garden are used as elements of spatial articulation, as functional spaces intended for the owner's use of the palace and to highlight the fame and prestige of the families interested in promoting their name over the restricted local borders.

KEYWORDS: Open Staircase, Episcopio di Sarno, Residence, Late Baroque, Ferdinando Sanfelice.

Recibido: 14 de abril de 2016 / Aceptado: 15 de septiembre de 2016.

L'uso della scala aperta nella residenza barocca e tardobarocca in Campania rappresenta uno dei temi che hanno maggiormente inciso sulla progettazione degli spazi privati tra Sei e Settecento (Cantone 2010: 13-41). Qui il tardobarocco registra elementi di contaminazione con gli ambienti liguri e con la cultura spagnola (Ruiz Barbarin, 2012; Marías, 2005), oltre che con la cultura mitteleuropea, i cui contatti furono favoriti dal periodo vicereale austriaco.

In area italiana prima, in ambito europeo poi, la definizione di macchine complesse dalle differenti geometrie ha giocato un ruolo di primo piano nella determinazione degli elementi costitutivi la residenza barocca. Attraverso i nutriti

GIUSTO, Rosa M.: "Architettura del Settecento a Episcopio di Sarno. La scala aperta nella residenza tardobarocca tra capitale e provincia", Boletín de Arte, n. ${ }^{\circ}$ 37, Departamento de Historia del Arte, Universidad de Málaga, 2016, pp. 89-101, ISSN: 0211-8483. 
scambi verificatisi tra Italia e Europa, tali linguaggi subirono modifiche e trasformazioni fino a confluire, determinandole, in diverse soluzioni architettoniche messe a punto nel XVIII secolo da artisti particolarmente aperti ai contatti e alle contaminazioni.

\section{Napoli e l'Europa}

A partire dal 1707 e fino al 1734, anno in cui Carlo III di Borbone avvia la costituzione di un regno indipendente, è il Meridione d'Italia e Napoli in particolare a conoscere un periodo d'intensa attività edilizia e di notevole slancio creativo: il ripetuto contatto con la realtà austriaca favorendo scambi tra l'ambiente partenopeo e la cultura danubiana, evidenti soprattutto in tema di architetture civili dove si determinano nuove soluzioni tipologiche e distributive (Garms, 2000; Labrot, 1993). All'interno di tale geografia, Napoli rappresenta senza dubbio il centro principale di irradiazione dal quale, innestandosi su di una tradizione già quattrocentesca, il tipo della scala aperta ebbe modo di consolidarsi e «raffinarsi» per raggiungere, nelle sperimentazioni architettoniche di Ferdinando Sanfelice, l'apice di una ricerca spaziale e linguistica le cui tracce numerose si ritrovano in diversi centri del territorio regionale fino a divenire uno degli elementi caratterizzanti la residenza tardobarocca in Campania (Nykjaer, 1993). Furono proprio Ferdinando Sanfelice (Gambardella, 1974; Ward, 1988) e Domenico Antonio Vaccaro (Gravagnuolo, 2005) a rappresentare il filone dell'architettura tardobarocca campana, rielaborando in modo originale temi insiti già nella tradizione costruttiva locale, contaminati con elementi desunti dalla 'vicina' Mitteleuropa, recepiti soprattutto per ciò che attiene agli apparati decorativi, fino a divenire due dei più prestigiosi protagonisti della stagione artistica partenopea a cavallo tra viceregno austriaco e dominazione borbonica. (Vásquez Gestál, 2012: 14-38).

Nel valutare la «contiguità» tra il linguaggio tardobarocco europeo e le opere di Domenico Antonio Vaccaro, Anna Maria Matteucci sottolineava come «in analogia con i coevi orientamenti artistici dei paesi cattolici di lingua tedesca, i bianchi motivi decorativi a stucco non sono contenuti in apposite zone ma, nel loro espandersi, tendono a nascondere le strutture fondamentali dell'edificio o a fondere elementi architettonici solitamente fra loro disgiunti» (Matteucci, 1988:
121; Blunt, 1975). Diversamente dal discorso atettonico di Vaccaro (Matteucci, 1988: 124), rintracciabile nella completa compenetrazione tra struttura e apparati decorativi tipica dell'alta stagione barocca, Ferdinando Sanfelice sperimenta una ricerca incentrata sugli elementi strutturali della fabbrica, ricondotta a «una semplice ma esibita intelaiatura» (Matteucci, 1988: 124).

La mutazione che l'organismo-scale subì da un ambito tipologico a un altro, nel passaggio tra Sei e Settecento, va letta proprio come trasfigurazione, all'interno dell'edificio civile, dello "scalone d'onore» delle residenze reali. Si pensi allo scenografico scalone innestato da Filippo Juvarra all'interno di palazzo Madama a Torino, o alla soluzione ideata da Lukas von Hildebrandt per il Belvedere Superiore di Vienna, o, ancora, allo scalone a doppia rampa contenuto in una gabbia traforata, completato da Jacob Prandtauer tra il 1709 e il 1710 nel monastero agostiniano di San Florian, in Austria, dove la disposizione della scala a gabbia aperta, più che la sua struttura tettonica, rivela un rapporto di «parentela» con talune soluzioni sanfeliciane (Divenuto, 2003: 148; Matteucci, 1988: 124). Queste, tuttavia, sperimentano una maggiore inventiva strutturale e compositiva adoperando il dispositivo della gabbia forata quale elemento «strutturale» della scala, prim'ancora che quale elemento linguistico. In altri termini, forma e funzione si fondono nelle soluzioni sanfeliciane: I'ossatura strutturale della gabbia contenente le scale coincidendo con l'intelaiatura dei rampanti e delle soste ai piani delle scale.

In relazione alla «mutazione tipologica» che interessò le residenze di città in quegli anni, il tema delle scale finì per assumere un duplice significato: da un lato, costituì uno degli elementi più raffinati in grado di documentare l'importanza, il censo e il prestigio della famiglia proprietaria dell'immobile; dall'altro, si ridimensionò e si contrasse per essere impiegato, sia pure in maniera semplificata, nei palazzi di civile abitazione, dalle residenze borghesi d'alto censo alle cosiddette case d'affitto che proprio Sanfelice a Napoli ebbe il merito, tra i primi, di delineare tipologicamente. Palazzo Serra di Cassano rappresenta, contemporaneamente, un modello di residenza privata di alto rango e uno dei primi esempi di edilizia condominiale cittadina, qualificato architettonicamente.

Alla luce delle profonde innovazioni introdotte in ambiente napoletano da Sanfelice, non sorprende l'influenza che l'elemento scale conobbe nell'ambito delle nuove tipologie abitative, divenendo di fatto il mezzo attraverso il quale fu 
garantita una più funzionale distribuzione degli appartamenti e una loro più agevole sistemazione, anche in ragione degli elementi di collegamento e di attraversamento comuni costituiti dai cortili. Fu proprio Ferdinando Sanfelice a innescare un serrato dialogo tra blocco scale e cortile. Ponendo in un preciso rapporto gerarchico-funzionale le due parti, giunse a declinare una sorta di «repertorio» delle possibili soluzioni tipologiche in cui la relazione scale/cortili veniva attentamente vagliata e sperimentata. A partire da uno degli elementi «canonici» dell'architettura civile e urbana partenopea -le scaleegli altera i rapporti tra i volumi, puntando l'attenzione sull'uso originale e personalissimo di quei vuoti urbani -i cortili- destinati ad assumere enorme rilevanza quali elementi di smistamento e di coagulo tra la strada, il vestibolo, gli ambienti interni al palazzo e il giardino (laddove presente) disposto oltre il corpo scale in asse con l'ingresso principale. II cortile e le scale divengono così i nodi attorno ai quali strutturare nuovi schemi distributivi, la maggior parte dei quali regolati da rinnovate gerarchie funzionali. Ci troveremo, così, di fronte a diversi tipi di scale: da quelle che, riprendendo il tema dello scalone monumentale, occupano un intero ambiente della fabbrica come nel già citato caso di palazzo Serra di Cassano dove essa abita addirittura un intero vano a doppia altezza; a quelle a "cannocchiale» o ad "ali di falco", ambedue impiegate nel palazzo Sanfelice ai Vergini dove cortili contigui, ma separati, ospitano scale dalle differenti tipologie. Anche in questo caso, scale e cortili vengono adoperati per distribuire e moderare $\mathrm{i}$ flussi d'ingresso al palazzo, generando un processo di addizione e d'incastro di volumi composti di differenti geometrie.

Le scale aperte napoletane rappresentano nella età barocca la più originale espressione della edilizia locale, che non trova riscontro in alcun'altra regione d'Italia. Nacquero nel '400 -si ritrovano già nel Palazzo che fu di Antonello Petrucci in piazza San Domenico Maggiore [...]- trovarono assertori audaci e convinti nel Sanfelice e in Domenico Antonio Vaccaro, che tradurranno il ritmo della arcate rinascimentali nella scenografica tensione delle crociere e degli archi rampanti, avvalendosi di geniali e libere invenzioni (Capobianco, 1962: 403).

Tra tali soluzioni, lo schema senza dubbio più replicato fu proprio quello della scala aperta ad ali di falco nella quale un'intera parete del cortile d'accesso al palazzo, solitamente quella frontale all'ingresso, è occupata in tutta la sua esten- sione e ampiezza da una gabbia forata contenente le rampe che determina una sorta di seconda facciata, assai più sontuosa ed eloquente di quella principale. Accade, infatti, che mentre esternamente è il solo portale a segnalare il grado di nobiltà e il prestigio della famiglia proprietaria dell'immobile -concentrandosi gli elementi decorativi nello stemma, nelle mensole, nei capitelli e in generale nel disegno dei dettagli architettonici-, internamente l'intera parete contenente le scale vive di un'autonoma e dinamica configurazione plastica e spaziale, tanto più evidente e significativa quanto più il contrasto con la semplicità degli esterni è marcato e «stridente». Questo aspetto è da collegarsi alla sensazione di stupore e meraviglia che l'estetica del barocco intese suscitare nell'osservatore, ma anche a quella discrasia tra interni ed esterni inaugurata proprio da quella stagione artistica, oltre che dal trasferimento di una "valenza urbana» all'interno di uno spazio privato -il cortile del palazzo. Cosicché, se è innegabile il legame che la scala aperta instaura con l'edificio, altrettanto evidente è la relazione ch'essa innesca con l'ambiente urbano circostante: tra strada, vestibolo, cortile e scala il sistema edilizio e quello urbano si confrontano in un rapporto di reciproca relazione dinamica fino a determinare il prolungamento dell'asse della strada nel cortile del palazzo. II saldo rapporto instaurato tra edificio e tessuto urbano è sottolineato proprio dalla presenza di un unico asse di attraversamento che, passando per il blocco mediano delle scale, mette in comunicazione visiva strada, corte interna e giardino retrostante. Questa successione di spazi in profondità risponde ad esigenze ancora una volta di tipo "scenografico", alludendo al tema dell'asse all'infinito, mentre l'asse trasversale del corpo-scale suggerisce una gerarchia di funzioni che culmina, nei casi più complessi, in una moltiplicazione dei cortili di cui il principale collegato ai secondari tramite proprio il blocco-scale.

Quanto più elegante e raffinato è il palazzo, tanto più gli spazi che precedono le scale si moltiplicano: superato il portale d'accesso si ritrovano, infatti, l'atrio, cui segue il vestibolo che apre sul cortile, in alcuni casi funzionando da elemento di connessione con le rampe della scala.

L'influenza esercitata da Sanfelice sul tema della residenza fu dovuta a una serie di fattori tra i quali, accanto alle richiamate circostanze politiche, anche quello d'essere stato allievo e collaboratore di Francesco Solimene, uno dei più stimati e autorevoli artisti attivi pressoché in tutti i cantieri più importanti del Meridione. Una diffusione che si verifica 
anche in ragione della consuetudine, invalsa tra le famiglie aristocratiche del tempo, di farsi edificare seconde abitazioni in cui trascorrere periodi di vacanza e di riposo lontani dai più affollati e caotici centri urbani. Basti pensare alle ville vesuviane, quel sistema di abitazioni, luoghi di delizie e tenute fuori città che, proprio a partire dal XVIII secolo, si andò «strutturando» secondo una caratterizzazione architettonica sempre più definita, tanto da comportare il delinearsi di veri e propri «modelli» di riferimento in tema di residenze suburbane dall'elevato carattere paesaggistico. In esse, l'inserimento delle scale "a giorno" sul cortile principale del palazzo quale sistema arioso ed elegante di collegamento verticale, divenne una pratica diffusa assumendo conformazioni tra le più ricercate, legate alla singolare morfologia della produzione architettonica facente capo proprio ai modelli tardobarocchi introdotti da Ferdinando Sanfelice e Domenico Antonio Vaccaro.

Analogamente, nelle aree vesuviane e nel salernitano ville e seconde dimore si arricchirono dei preziosi sistemi verticali, sull'esempio delle soluzioni realizzate in ambito partenopeo. Non stupisce, pertanto, constatare come espedienti progettuali adoperati a villa Meola a Portici, a villa Vannucchi a San Giorgio a Cremano, a villa Granito di Belmonte a Ercolano, siano poi «migrati» nelle vicine località dando luogo a soluzioni più semplici e contenute, ma non per questo meno significative sotto il profilo del linguaggio architettonico e dei caratteri distributivi impiegati (Garms, 2000: 279-282; De Seta, Di Mauro, Perone, 1980: 29).

\section{La scala aperta a Episcopio di Sarno}

Nel valutare la fortuna e l'impatto che i sistemi verticali sanfeliciani riscossero in ambito campano, Anna Maria Matteucci citava quali immediati riferimenti palazzo Conforti e palazzo Ruggi d'Aragona a Salerno, nonché una serie di «edifici e palazzetti» a Episcopio di Sarno che ne costituirebbero suggestive filiazioni (Matteucci, 1988: 130).

In particolare, nella Valle del Sarno la residenza tardobarocca si carica di ulteriori influenze derivanti dalla presenza a Napoli di importanti famiglie -alcune delle quali di origine genovese- che, radicatesi da tempo in città, dove si erano fatte costruire imponenti palazzi e dimore, contribuirono a diffondere quei modelli abitativi anche nei propri feudi di pro- vincia, fungendo da elemento propulsore per la diffusione dei linguaggi artistici dalla capitale alle «periferie» del regno.

Sulla incidenza che i modelli liguri esercitarono sulla cultura architettonica del periodo e sugli sviluppi del tema della scala aperta basti ricordare il ruolo svolto dal cinquecentesco Palazzo Doria Tursi di Genova; I'edificio divenne rapidamente un modello di residenza privata di alto rango che, con il suo arioso e imponente sistema di scale e di logge, col suo succedersi di atrio, scala, cortile soprelevato e scalone a doppia rampa, è stato a giusta ragione ritenuto un elemento nodale nel processo di trasmissione e di codificazione di tale tipologia architettonica tra centri e periferie del Mediterraneo. Peraltro, proprio i Doria, principi di Angri dal 1636, costituiscono un importante trait-d'union tra la cultura ligure di origine e quella partenopea di «adozione», dove risale alla metà del '700 l'erezione del Palazzo Doria d'Angri, uno dei più sontuosi e rappresentativi edifici privati affidati alle cure di alcuni tra i più rinomati architetti del tempo. La presenza dei Doria nella valle del Sarno, di cui divennero duchi di Eboli e conti di Capaccio, è il segno evidente dell'insediarsi nell'Agro-Nocerino Sarnese di un sistema feudale di derivazione cittadina destinato a influenzare attivamente le politiche di espansione urbana.

Accanto ai Doria d'Angri, infatti, si registra la presenza di un cospicuo numero di famiglie appartenenti alla nobiltà cittadina alcuni delle quali, come i Tuttavilla o i Normandia, di origini francesi, altre legati alla nobiltà locale come gli Alteda, gli Ungaro, gli Abignente che richiamarono al loro seguito artisti tra i più prestigiosi e qualificati del tempo impegnati nei lavori di edificazione e ristrutturazione dei possedimenti aviti o nell'edificazione di cappelle gentilizie all'interno dei palazzi e delle chiese, determinando un clima di operosità e crescita urbana (Labrot, 1979).

Ricostruendo il fitto intreccio di relazioni famigliari legate all'espansione del feudalesimo nella valle del Sarno è possibile risalire ai canali attraverso i quali si verificò la diffusione del sistema delle scale aperte all'interno delle abitazioni private di Episcopio, influenzate da un analogo processo di «metamorfosi» della residenza tardobarocca, oltre che dall'attività di maestri quali lo stesso Sanfelice, Domenico Antonio Vaccaro, Angelo e Francesco Solimene. II quadro che emerge è di grande interesse per una più estesa indagine conoscitiva del territorio campano dove si ritrovano edifici tardobarocchi inediti nei quali la tipologia delle scale aperte e 


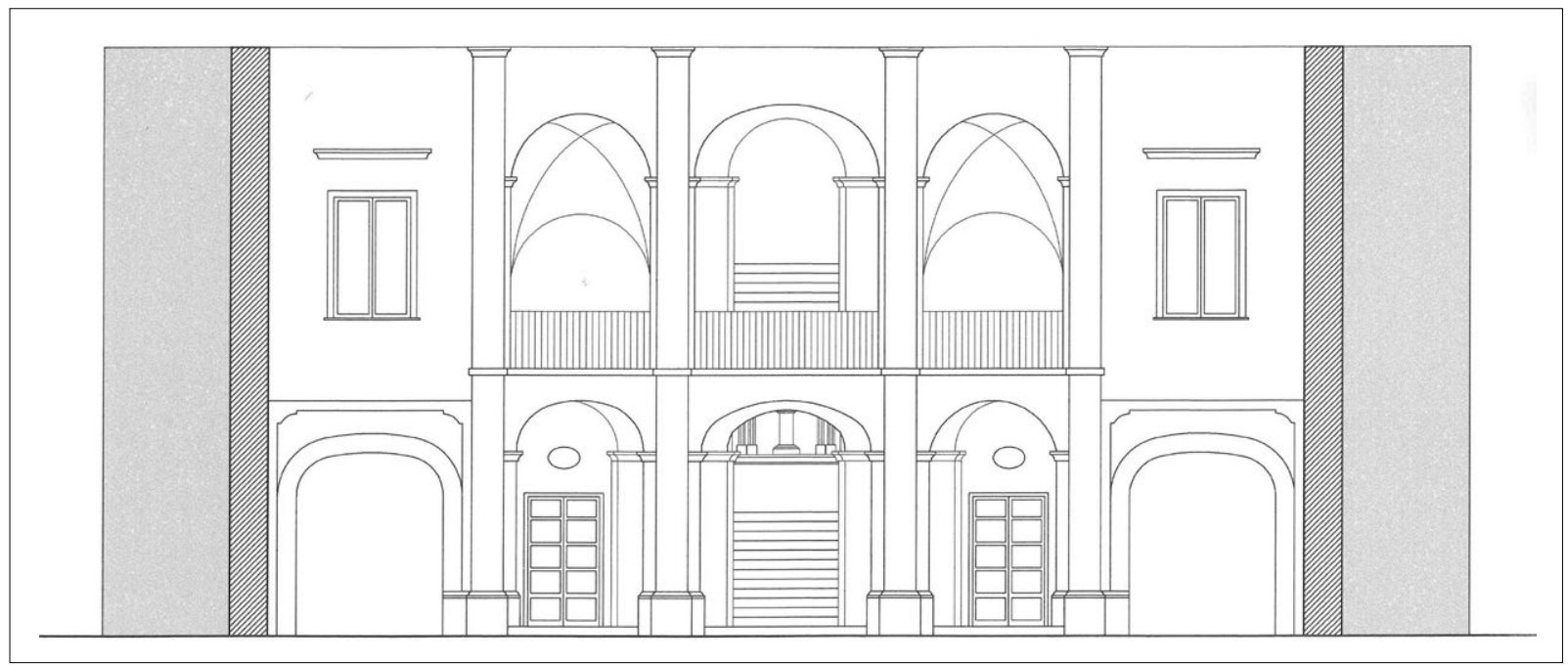

la sua connessione col cortile e col giardino retrostante funge da elemento di articolazione spaziale e distributiva degli ambienti, oltre che da spazio «funzionale» destinato agli usi padronali del palazzo.

Proprio su Episcopio di Sarno è opportuno soffermare l'attenzione dal momento che la piccola frazione fu, dall'XI secolo, sede vescovile. Tale circostanza, unitamente all'intensificarsi degli scambi e dei commerci, favorì l'affermarsi in età moderna di una solida economia imprenditoriale avallata dagli stessi feudatari del luogo che ne promossero lo sviluppo economico e sociale. Durante il Sei e il Settecento, come per altre località, anche Episcopio subì notevoli trasformazioni: le modeste case rurali sorte nei pressi della cattedrale lasciarono il posto a palazzi signorili, molti dei quali caratterizzati da una tipologia a corte con scala aperta e giardino retrostante.

La scelta di far erigere la nuova cattedrale su di un'area demaniale non soggetta alla giurisdizione feudale aveva comportato l'edificazione della chiesa e del successivo palazzo vescovile in un sito distante dal centro abitato di Sarno. La cattedrale venne infatti edificata su di un'aera collinare attorno alla quale andarono distribuendosi le ricche famiglie latifondiste della zona interessate a godere delle franchigie concesse all'alto clero e ai monasteri.

Politicamete Salerno dipendeva dal viceregno, ciò spiega la presenza di Sanfelice in diverse località della pro-

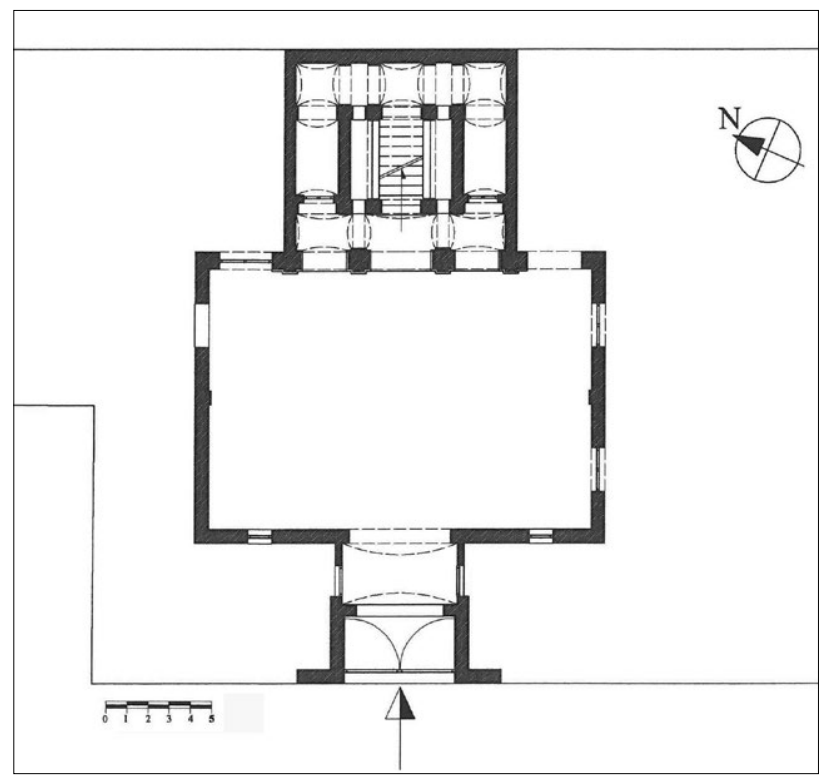

1. Episcopio di Sarno, palazzo Origo in via Duomo n. ${ }^{0} 133$, pianta e prospetto della scala aperta. Elaborazione grafica Marika Monaco

vincia, dove lavorarono intensamente anche Angelo e Francesco Solimene, originari del nocerino, con i quali Sanfelice collaborò stabilmente in quel progetto di sintesi delle arti che accomunò entrambe le «scuole». Testimonianze eloquenti di tale attività sono documentate dal cassettonato della cattedrale di Episcopio e dalla cona d'altare, progettate e decorate da Angelo e Francesco Solimene. La collaborazione tra i Solimene e Sanfelice contribuì a determinare il formarsi di 


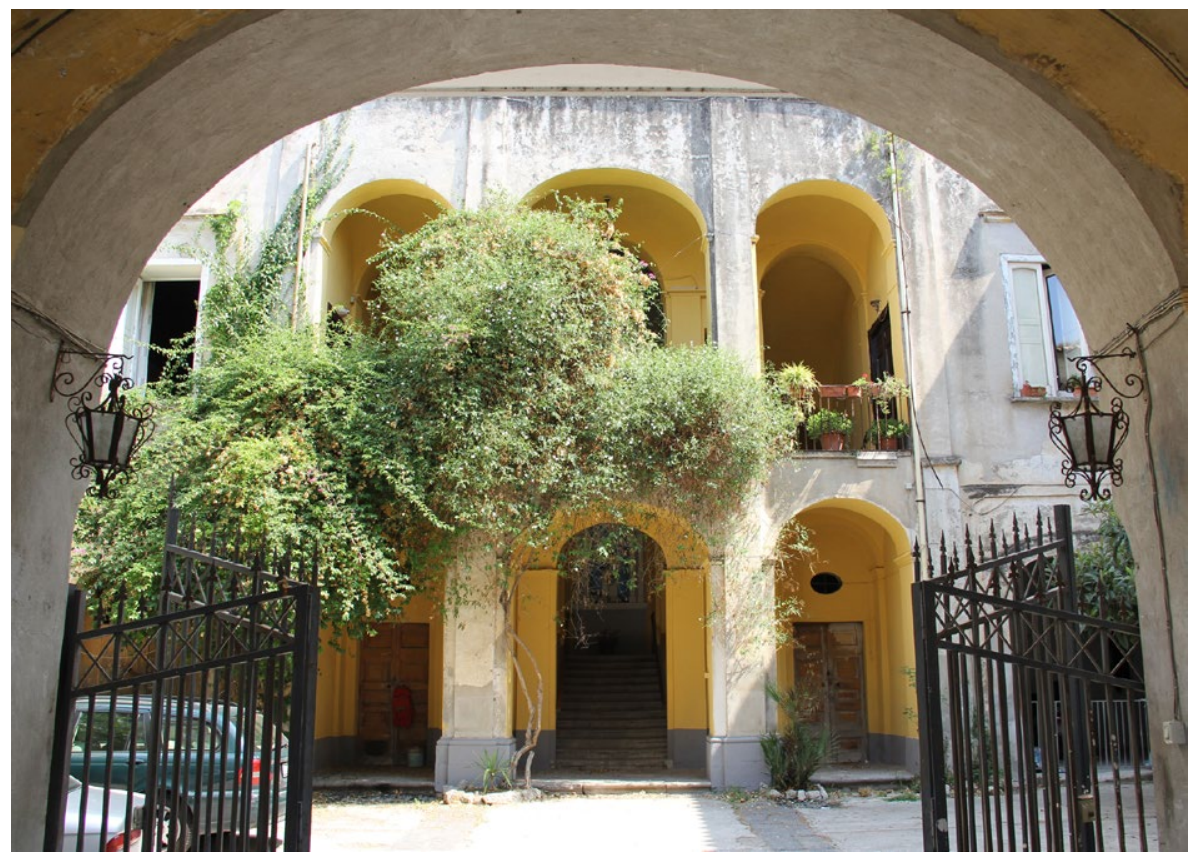

2. Episcopio di Sarno, palazzo Origo, veduta della scala aperta dal vestibolo d'ingresso al cortile (fotografie dell'autrice)

una professionalità diffusa tra le maestranze locali, in grado di replicare, non senza qualche ingenuità, il linguaggio più volte sperimentato dai maestri. Si spiegano così i numerosi esempi e variazioni di scale aperte settecentesche presenti nel piccolo borgo sarnese, che durante il XVIII secolo registrò un notevole impulso edilizio. I modelli di residenza privata affermatisi nei centri maggiori si diffusero rapidamente anche a Sarno dove si arricchirono della componente paesaggistica, caratterizzata da vaste aree verdi a completamento delle abitazioni e da vedute aperte sul Vesuvio e sul golfo di Napoli. Tra essi si segnalano alcuni edifici nel centro antico di Episcopio che, per la loro singolarità rispetto al tessuto urbano circostante e per l'evidente filiazione dai modelli partenopei, meritano di essere trattati singolarmente. La loro collocazione prevalentemente lungo l'asse di via Duomo, che attraversava longitudinalmente la frazione di Episcopio congiungendo il centro di Sarno con la cattedrale, denota il censo delle famiglie proprietarie degli immobili che intesero farsi costruire le proprie residenze secondo i modelli in uso a Napoli e nella vicina Salerno.

La graduale sostituzione dell'antica nobiltà locale con la nuova e più solida nobiltà cittadina e di provincia aveva, infatti, comportato il determinarsi di condizioni politiche ed economiche favorevoli allo sviluppo edilizio del borgo, particolarmente florido a partire proprio dai primi anni del Settecento. Le famiglie notabili stabilitesi a Episcopio si impegnarono a far fruttare i propri suoli impiegandoli per la produzione agricola. I loro nomi compaiono nelle iscrizioni delle cappelle laterali del duomo dove troviamo documentata la presenza dei Barbaroli, degli Origo, dei Milone proprietari di alcuni tra i palazzi più interessanti e aggiornati stilisticamente sorti a Episcopio di Sarno.

Percorrendo via Duomo, al civico 133, sorge il palazzo degli Origo [1]. L'abitazione presenta una tipologia a corte chiusa rettangolare con androne d'accesso voltato a botte e scala aperta frontale che serve un unico livello. Qui l'apparato della scala non occupa per intero il lato frontale all'ingresso, ma soltanto la sua porzione intermedia, di forma quadrangolare, lasciando un'eguale campata per lato occupata al piano terra dai locali originariamente destinati a frantoio e a depositi, con al primo piano due appartamenti disposti simmetricamente. Trattandosi di una costruzione con annesse funzioni rurali posta all'ingresso di via Duomo, dal lato opposto della 
3. Episcopio di Sarno, palazzo in via Duomo n. ${ }^{\circ}$ 91, pianta e prospetto della scala aperta.

Elaborazione grafica Marika Monaco

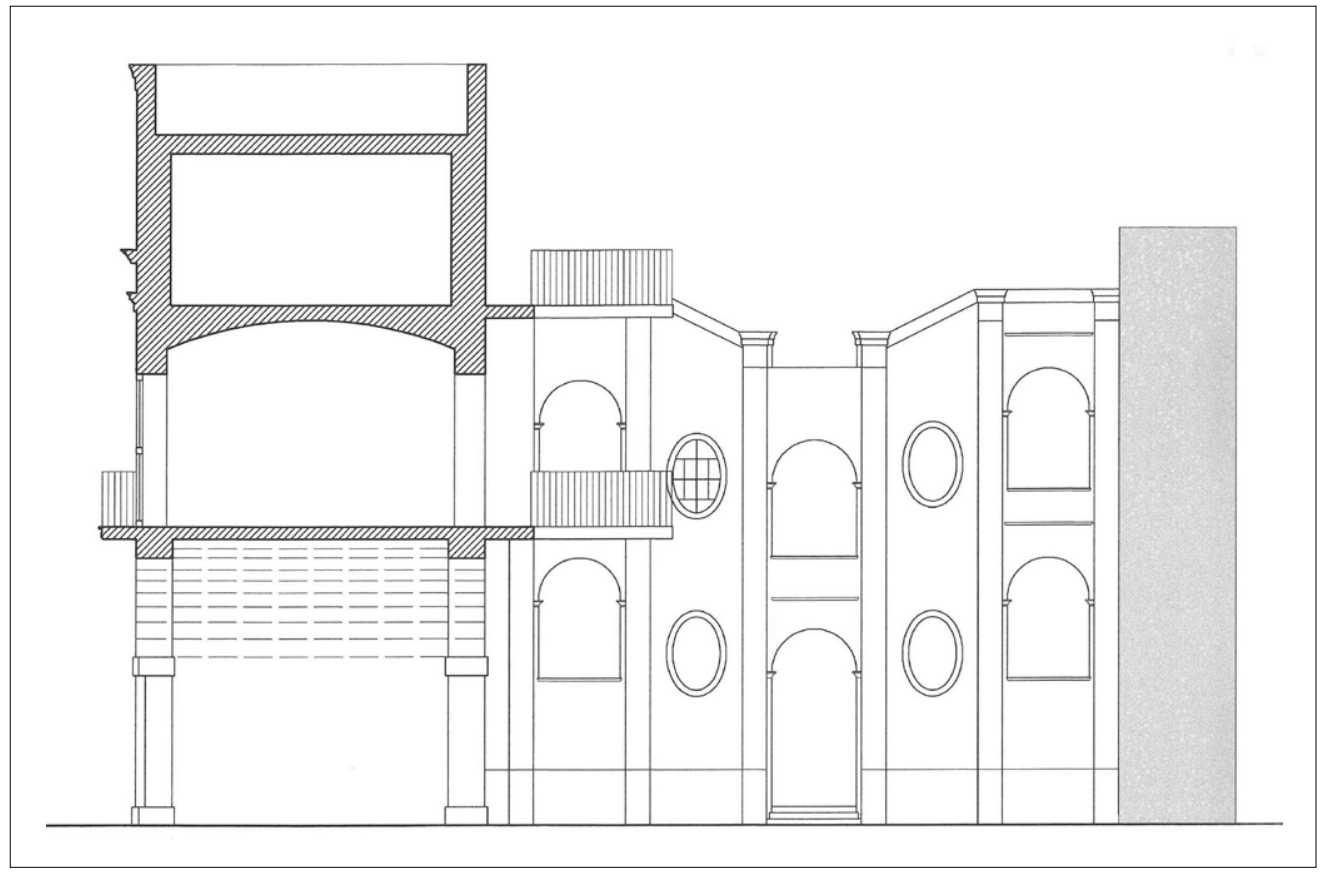

cattedrale, l'edificio ospitava al piano terra gli ambienti per la produzione e conservazione del raccolto e al primo piano l'abitazione del proprietario.

II prospetto della scala aperta [2] è composto di tre archi di cui quello centrale, più ampio, ribassato e i laterali a tutto sesto sorretti da pilastri su alte basi a cui sono addossate semplici paraste dai capitelli appena accennati coincidenti con la fascia marcapiano. Le coperture del portico, del pianerottolo intermedio e della loggia al primo piano sono realizzate con volte a vela, mentre le rampe sono coperte da volte a crociera rampanti. La tipologia della scala è a pozzo su doppi pilastri: una rampa unica s'innalza dall'arcata centrale raggiungendo un pianerottolo intermedio da cui si diramano due rampe laterali di minore ampiezza che immettono nella loggia al primo piano dove nasce l'ultima rampa centrale conducente al terrazzo.

Particolarmente degno di nota è il sistema strutturale della scala il quale impiega l'artifizio di «svuotare», con delle tese bucature rettangolari, il prospetto interno della scala, sia anteriormente che posteriormente, lungo le parti di muratura che campiscono lo spazio tra la rampa centrale e le laterali,

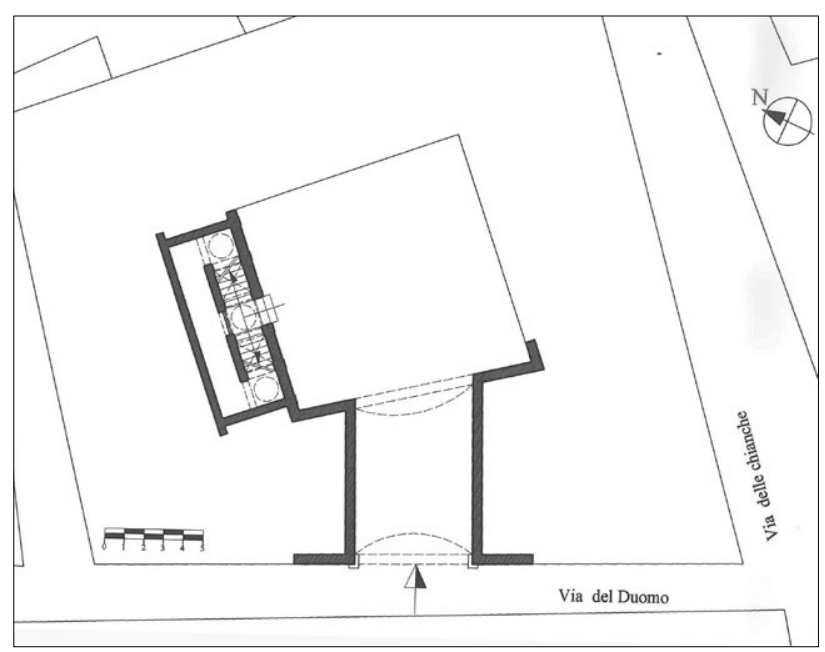

configurando un sistema interno della scala scattante e dinamico segnato dalle porzioni di muro assottigliate, dal gioco interno delle rampe e dei ripiani, dall'intersecarsi di archi a tutto sesto e a collo d'oca, determinando «pozzi di luce» e «Svuotamenti» verticali secondo un trattamento che ricorda, sia pure con ben altre implicazioni spaziali, quello adottato nel palazzo Trabucchi di Napoli, dove le bucature tra i pilastri sono trattate ad arco. 


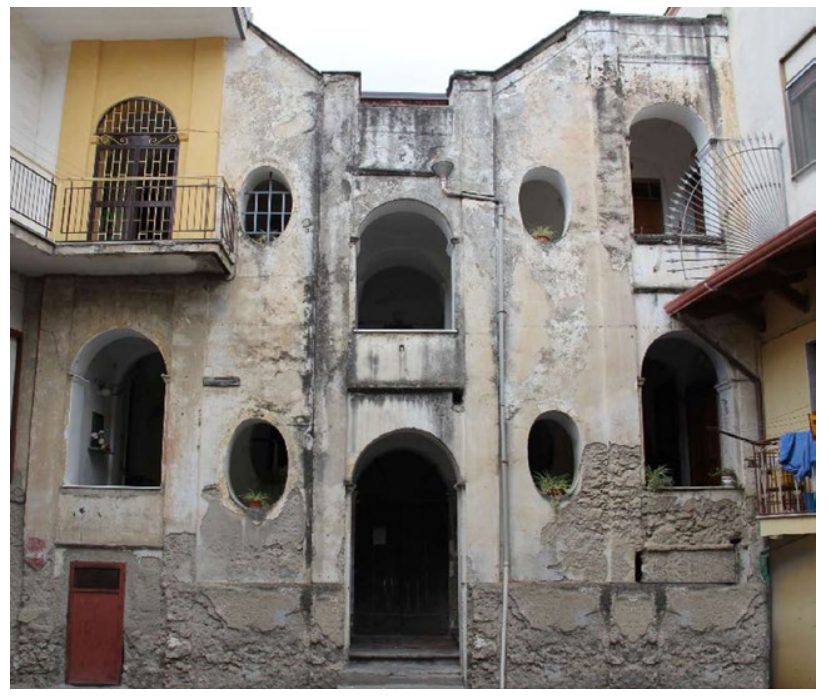

4. Episcopio di Sarno, palazzo in via Duomo n. ${ }^{\circ}$ 91, veduta della scala aperta dal cortile (fotografie dell'autrice)

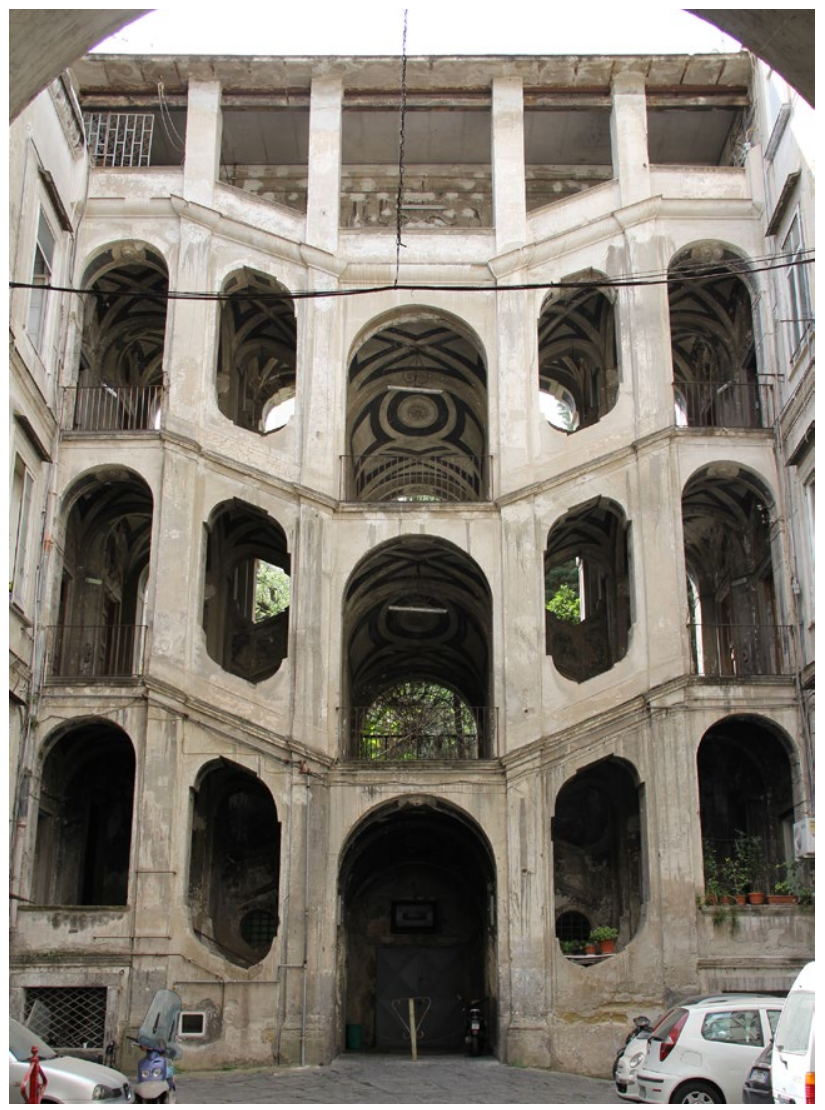

5. Napoli, palazzo Sanfelice ai Vergini, veduta della scala ad «ali di falco» dal cortile (fotografie dell'autrice)
Il prospetto esterno del palazzo, di recente restaurato, mostra con chiarezza il graduale ampliarsi dell'edificio da un primo impianto a tre campate, organizzato intorno al cortile centrale, ad ampliamenti successivi conseguenti alla progressiva annessione di nuovi vani. Di fronte al palazzo, sul lato opposto di via Duomo, in asse con l'ingresso principale, si apre un portale concluso da elementi in pietra e chiuso da un cancello su cui è riportata la scritta «Villa Origo» che immette nei vasti possedimenti terrieri posti a ridosso dell'abitazione padronale. La famiglia Origo, infatti, è annoverata tra le prime a insediarsi nella frazione di Episcopio decretandone lo sviluppo; tra i suoi discendenti si annoverano alti prelati e influenti personaggi, come attesta anche la lapide interna e come documenta lo stemma di famiglia raffigurato sulla volta dell'androne d'ingresso alla corte.

Proseguendo lungo via Duomo, al civico 91, s'incontra un palazzo dalla tipologia a corte con androne d'accesso voltato a botte e scala aperta posta lateralmente [3].

L'edificio occupa un'intera insula e, sebbene mal conservato, presenta ancora leggibile l'impaginato di facciata composto, su strada, di un piano terra trattato a basamento e di un primo piano con aperture a balconi contrassegnate da timpani semicircolari e triangolari alternati separati da lesene che sorreggono la trabeazione di coronamento e che definiscono un ritmo di cinque campate. L'impaginato a doppio ordine di lesene e aperture a timpano prosegue sui cantonali arrotondati del palazzo. Nel piano basamentale si aprono il portale d'accesso in pietra calcarea e quattro grandi finestre lobate. L'androne immette nel cortile quadrangolare il cui accesso irregolare «nasconde" la parete sinistra contenente il corpo scale. In tal modo, soltanto superato il vestibolo d'accesso ed entrati nello spazio aperto del cortile ci si accorge della presenza di un'intera parete laterale, orientata a sud, trattata a scale, il cui prospetto in alto è sagomato secondo l'inclinazione dei rampanti retrostanti, sottolineato dal ritmo diverso delle aperture e dalla trama delle cornici orizzontali che segna la posizione dei pianerottoli ai piani e le soste intermedie, secondo una soluzione analogamente impiegata (rampe finestrate, logge laterali a tutto sesto, coronamento finale) proprio da Ferdinando Sanfelice nel suo sontuoso palazzo di famiglia ai Vergini [4 e 5], oltre che nei palazzi Fernandes e Moscati o dello Spagnuolo a Napoli.

La scala riprende, infatti, semplificandolo, il modello sanfeliciano ad ali di falco. La soluzione adottata è costi- 


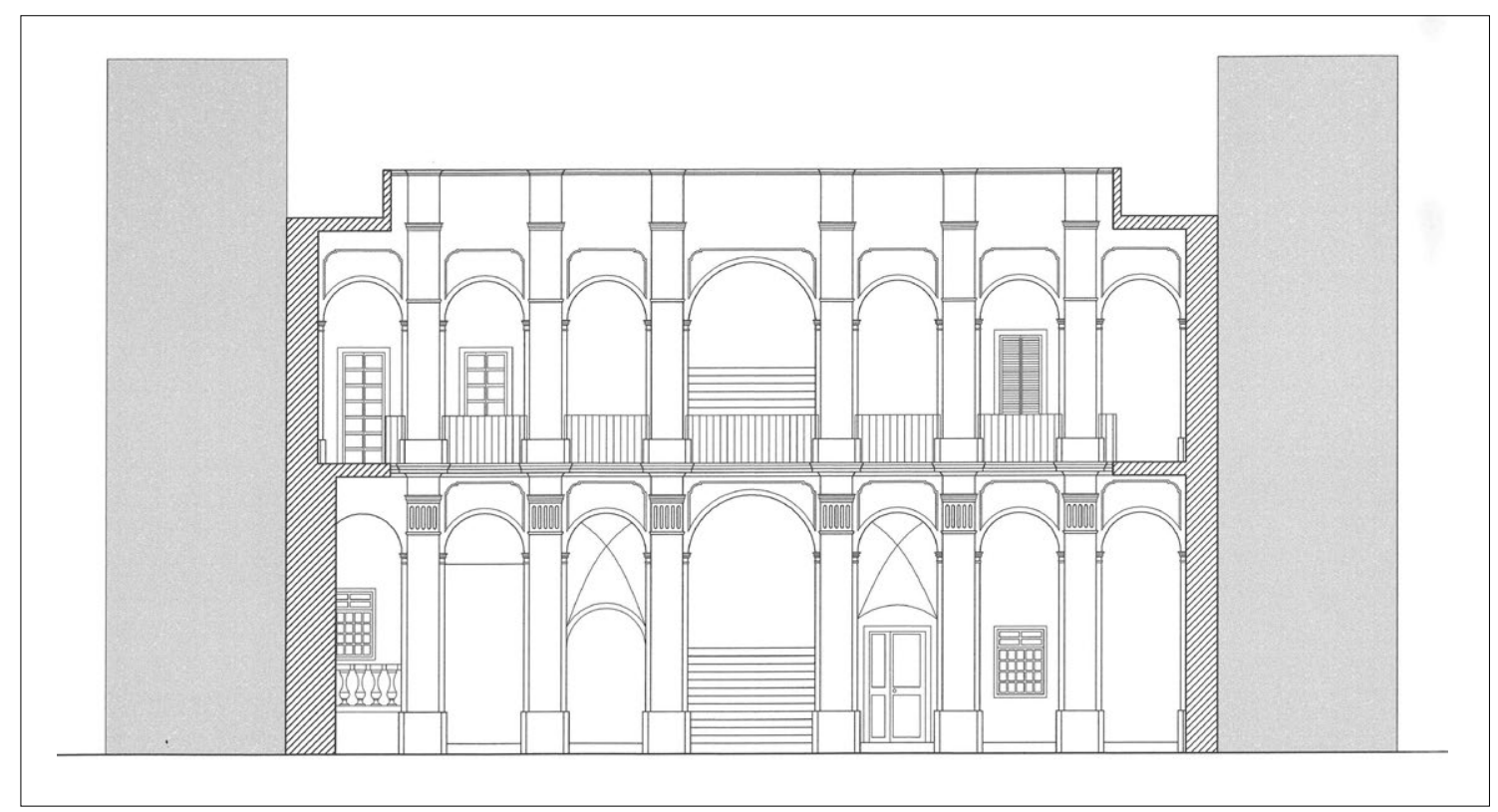

tuita da un pianerottolo centrale d'invito, rialzato rispetto al piano del cortile, da cui di diramano due rampe laterali che servono, su pianerottoli distinti, gli appartamenti disposti simmetricamente. Le due rampe si ricongiungono al livello superiore su di un unico pianerottolo centrale, in asse con quello di partenza.

Sei tese lesene giganti, concluse da scarni capitelli, contrassegnano le cinque campate di base che compongono la gabbia delle scale conducenti al terrazzo di copertura. Esternamente, il prospetto della scala presenta aperture ovali in corrispondenza dei rampanti laterali simmetrici, riprese anche sulle pareti interne, e aperture ad arco in corrispondenza dei pianerottoli di sosta. Questi sono coperti da «statiche» volte a vela sferiche sostenute da paraste i cui capitelli, in corrispondenza delle rampe sormontate da scattanti volte a crociera impostate su archi rampanti, seguono l'andamento obliquo, parallelo al verso delle scale. Come per analoghi casi napoletani, infatti, le volte che sovrastano e sostengono i pianerottoli sono costituite da «piccole volte sferiche ribassate sui piedi di volta» (Capobianco, 1963: 700), mentre le crociere delle rampe possono essere «considerate quali volte rampanti a collo d'oca [...] con la decorazione [che] determina l'effetto di crociera» (1963: 700).

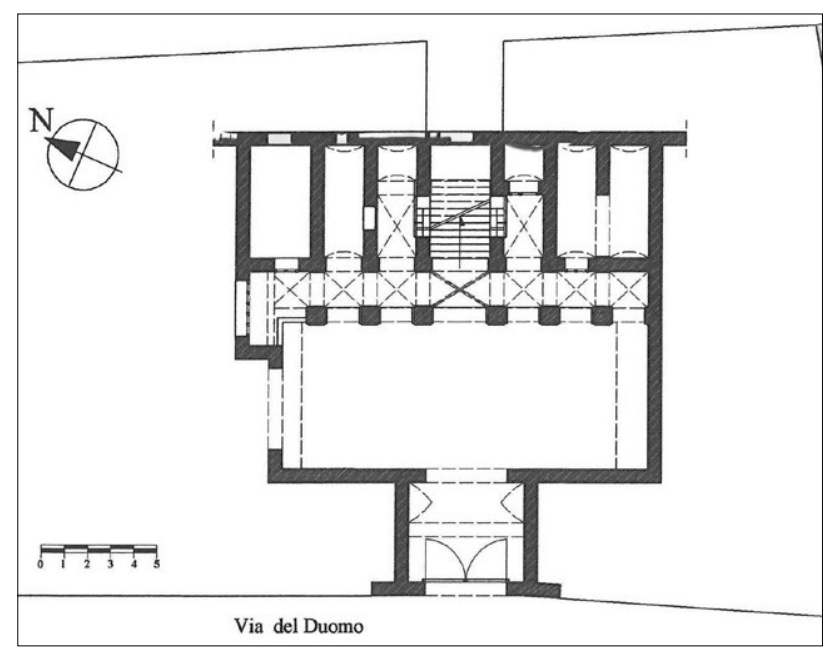

6. Episcopio di Sarno, palazzo Milone in via Duomo n. ${ }^{0} 101$, pianta e prospetto della scala aperta. Elaborazione grafica Marika Monaco

La gabbia delle scale guarda verso valle ed è concepita come affaccio e punto di vista privilegiato verso il Vesuvio e il golfo di Napoli.

Adiacente al palazzo di via Duomo, sorge palazzo Milone [6], appartenente all'antica famiglia il cui nome è documentato nella terza cappella di sinistra del duomo, partendo dal coro. L'edificio presenta una tipologia a corte chiusa con 


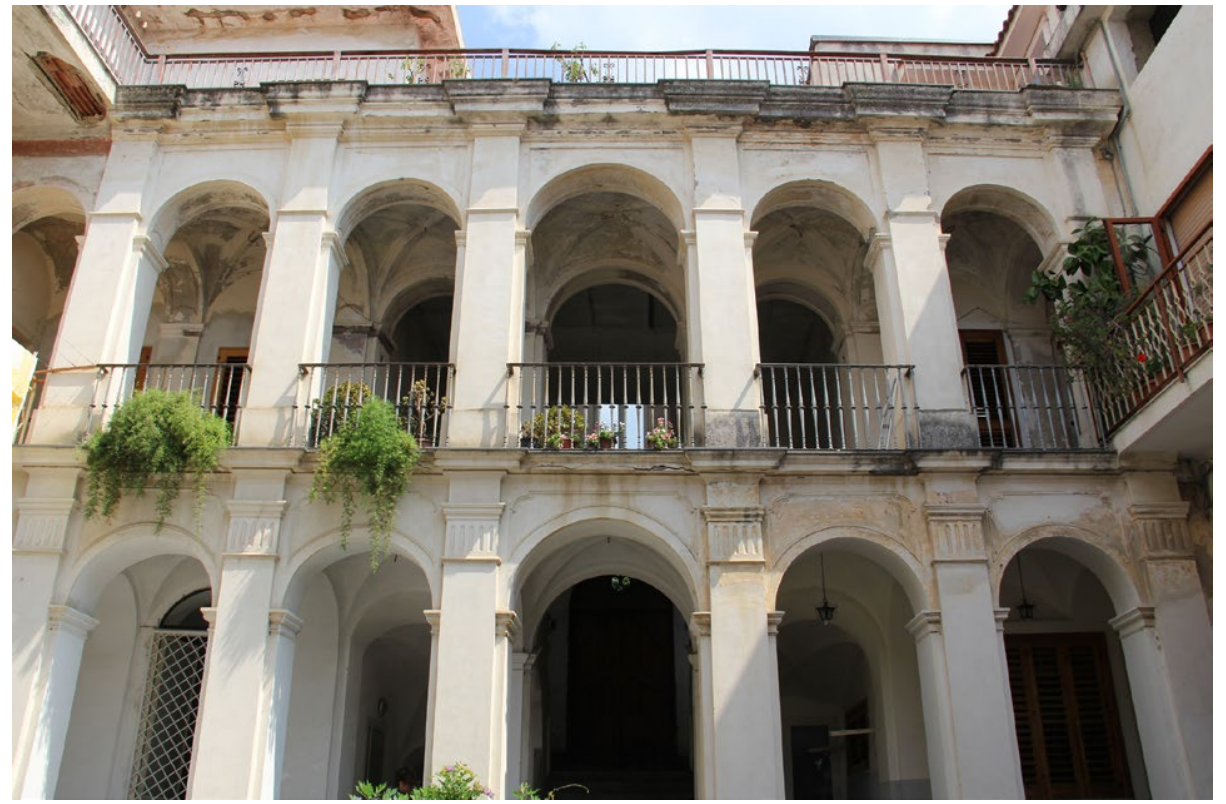

7. Episcopio di Sarno, palazzo Milone, veduta della scala aperta dal cortile (fotografie dell'autrice)

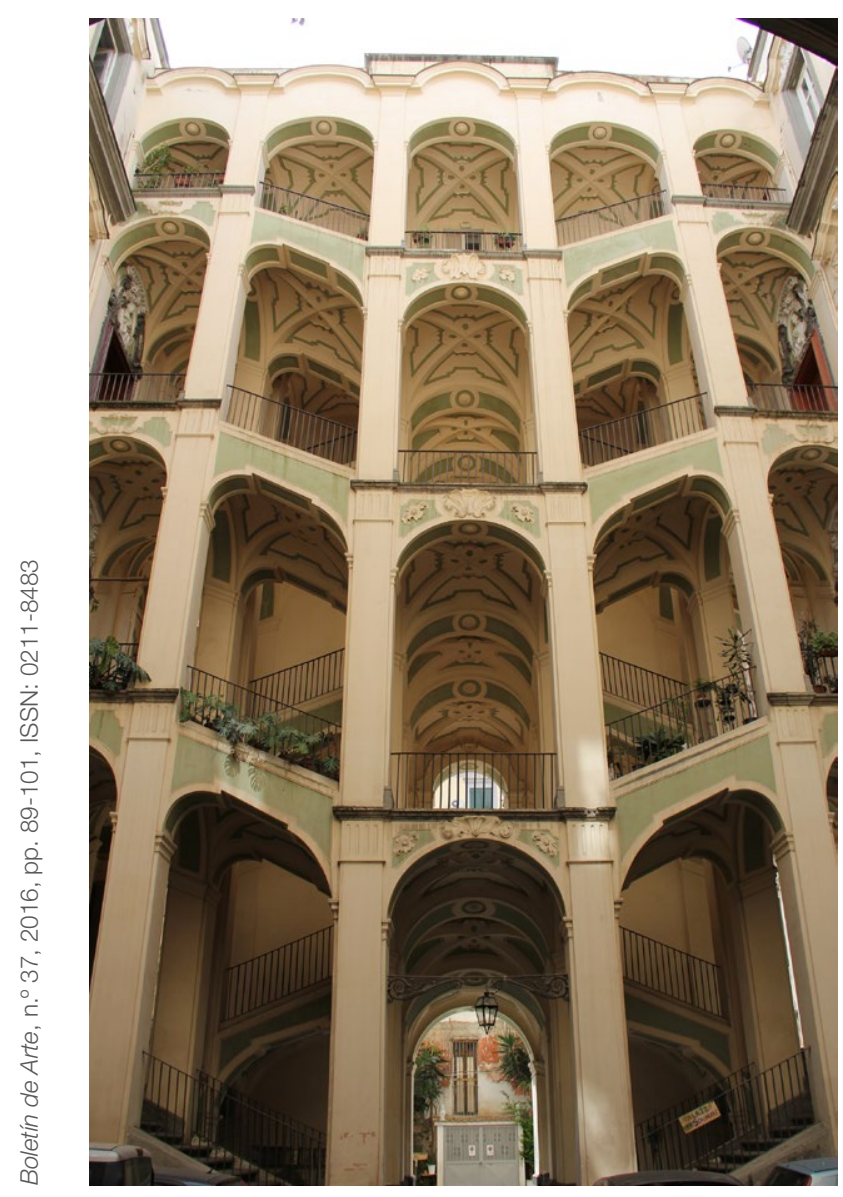

accesso dall'androne centrale coperto con volta a botte unghiata decorata a stucco.

L'edificio doveva essere particolarmente imponente se si considera che era articolato secondo una tipologia ad ali laterali simmetriche rispetto all'asse centrale di attraversamento collegate tre loro mediante il cortile rettangolare e la sontuosa scala aperta.

Tale impostazione può leggersi ancora sul prospetto principale lungo via Duomo, dove sono ancora perfettamente leggibili i blocchi simmetrici dell'edificio suddivisi in due campate per lato articolate al primo piano da balconi con timpani, fasce marcapiano, capitelli pensili e decorazioni in stucco dalle forme dichiaratamente rococò, e nel corpo centrale del cortile rettangolare, contrassegnato in facciata, per tutta la sua ampiezza, da una terrazza che corre sopra il portale d'ingresso denunciando anche all'esterno il «vuoto» del cortile concluso internamente dal loggiato aperto della magnifica scala sanfeliciana articolata in sette arcate rispetto

8. Napoli, palazzo Moscati o dello Spagnuolo ai Vergini, veduta della scala ad «ali di falco» dal cortile (fotografie dell'autrice) 
9. Episcopio di Sarno, palazzo Bosco in via Casamonica, pianta e prospetto della scala aperta. Elaborazione grafica Marika Monaco

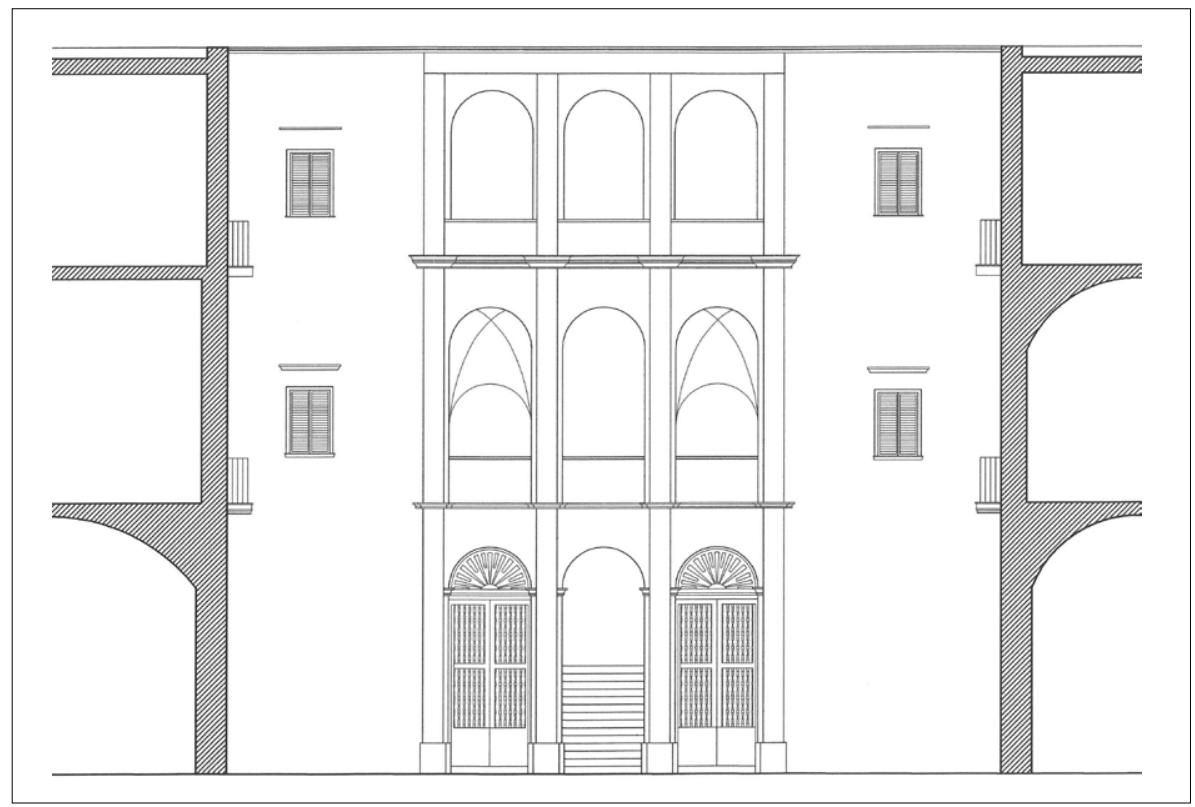

alle cinque delle pur più elaborate e complesse «macchine» partenopee.

Dall'androne centrale si accede al cortile rettangolare, dilatato lateralmente, il cui prospetto frontale è interamente occupato dalla grande scala aperta tardobarocca articolata su due livelli che, nonostante le trasformazioni subite dall'immobile, conserva intatta la sua tipologia. II prospetto della scala è composto a piano terra da sette campate contrassegnate da archi a tutto sesto, di cui il centrale più ampio; gli archi sono sorretti da pilastri che poggiano su alte basi in pietra [7]. Ai pilastri sono addossate piatte lesene concluse da capitelli scanalati la cui decorazione è identica a quella adoperata nel napoletano palazzo dello Spagnolo [8], motivo che induce a riflettere anche sulla possibile attribuzione dell'edificio; le arcate sono completate da decorazioni a stucco e dalla cornice marcapiano. Al secondo piano, il disegno si ripete pressoché identico con l'aggiunta del cornicione di coronamento. Le volte del portico al pian terreno sono del tipo a crociera, con il vano centrale coperto a crociera allungata decorata con nervature in stucco; tale decorazione si ritrova anche nelle volte della loggia al piano superiore.

La scala è composta di una prima rampa centrale, contenuta tra due muri intervallati da grandi aperture sagomate, che smonta su di un pianerottolo intermedio dal quale si accede al giardino retrostante rialzato disegnato all'italiana attorno

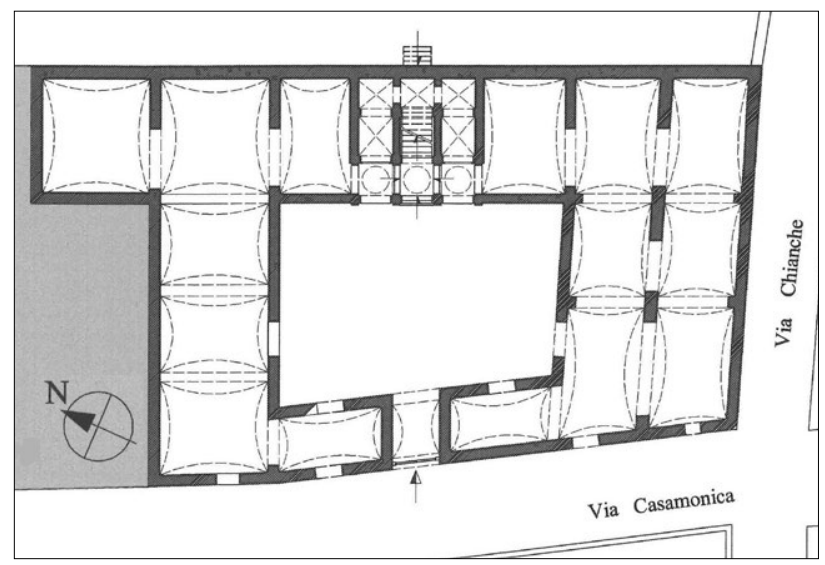

a un asse longitudinale centrale di attraversamento. Al termine del primo rampante, due raffinate mensole a volute completano i sostegni della scala. Da qui, a una quota di poco superiore, la scala di sdoppia in due rampe laterali, di ampiezza minore, che immettono nella loggia del primo livello da cui parte una rampa unica centrale conducente al pianerottolo intermedio con affaccio sul giardino e da lì al terrazzo di copertura.

Proprio l'accidentata orografia dei suoli ha comportato significative variazioni negli schemi distributivi adottati nelle residenze di Episcopio: il giardino non essendo quasi mai alla stessa quota del cortile, alle spalle della scala, ma, invece, rialzato rispetto al piano d'ingresso, escludendo l'articolazione 


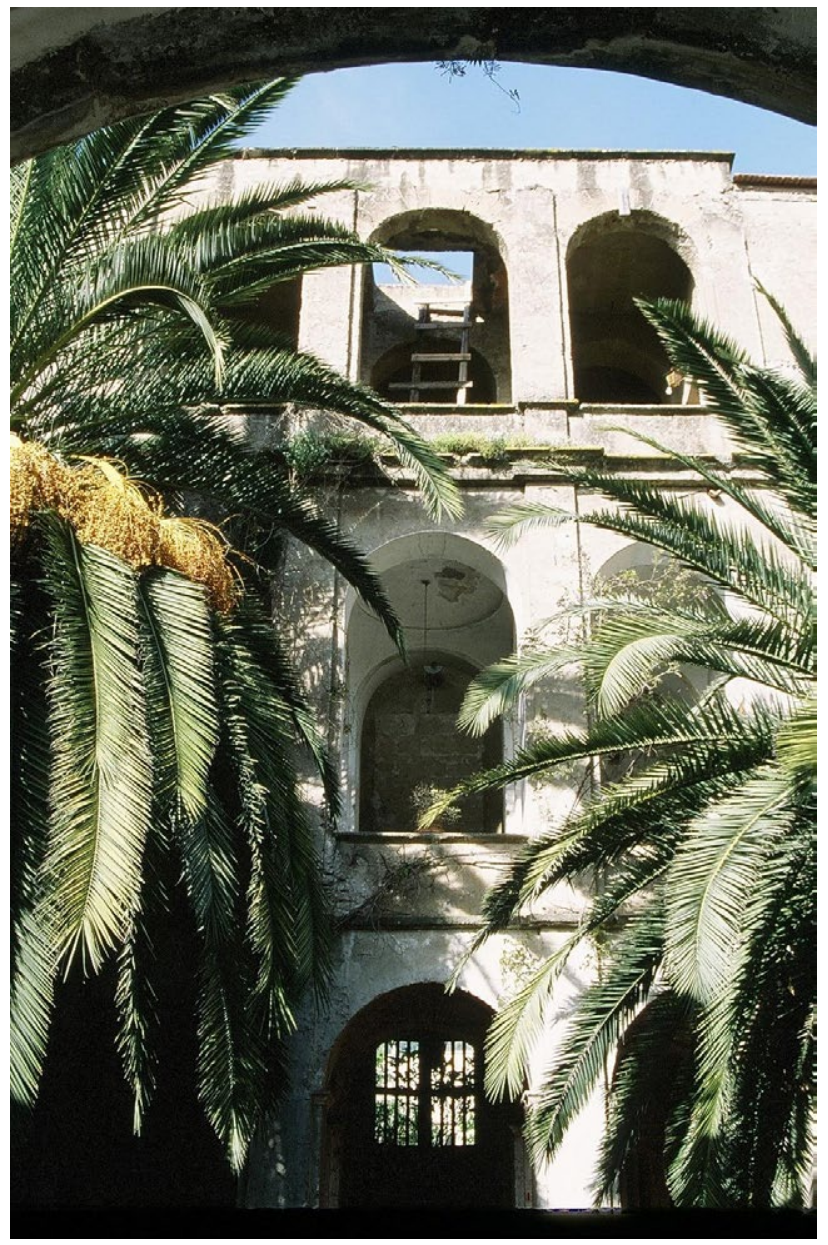

10. Episcopio di Sarno, palazzo Bosco, veduta della scala aperta dal cortile (fotografie dell'autrice)

"passante» al piano terra del vano scale, non più attraversabile in profondità.

Inoltre, essendo le rampe perpendicolari al prospetto della scala, le partiture architettoniche in facciata non seguono l'andamento obliquo delle rampe, come nei più suggestivi esempi napoletani, ma, tanto le cornici del primo piano, quanto le ghiere degli archi, presentano una struttura recta.

Un ulteriore elemento di interesse è dato dal rapporto tra il blocco scale e i corpi laterali adibiti ad abitazione. ॥ cornicione di chiusura del prospetto della scala, infatti, poggia sulle sole sei lesene che individuano le cinque campate centrali del loggiato, lasciando prive di coronamento la prima e l'ultima arcata. La volontà di trattare diversamente I' «ag- gancio» laterale della scala ai corpi trasversali dell'edificio è dichiarata proprio dall'assenza, alle due estremità, della lesena conclusiva e del relativo capitello: le arcate finali essendo «addossate» direttamente ai muri laterali.

Poco distante da via Duomo, lungo via Casamonica, sorge palazzo Bosco, con annessa la cappella gentilizia di cui è visibile esternamente il portale in pietra. L'edificio, d'impianto seicentesco, fu fatto erigere da Francesco Bosco (Astarita, 2003: 25) in un'area sottoposta al sito sul quale sorgeva la cattedrale, in segno di devozione. II palazzo ha conservato la planimetria originaria a corte chiusa con l'originario prospetto su strada composto di un terrazzo centrale che collega al primo piano le due ali laterali, secondo un'impostazione già riscontrata nel palazzo Milone [9].

Attraverso un androne voltato a botte si accede alla corte interna composta, al pian terreno, dei locali originariamente destinati alla produzione del vino e alla conservazione dei prodotti e con un pozzo; in asse con l'ingresso principale, si eleva un'imponente scala aperta composta di tre arcate a tutto sesto disposte su tre piani. La scala dà accesso al piano nobile dov'era l'abitazione del proprietario, con gli ambienti di rappresentanza decorati da affreschi, mentre il secondo piano era destinato alla servitù.

La scala si sviluppa nell'arcata centrale con un'unica rampa coperta da volta a crociera rampante che immette su di un pianerottolo intermedio da cui si ha accesso al vasto giardino retrostante, un tempo assai più esteso, che insieme al palazzo occupa l'intera insula con accesso anche dall'attuale viale Giovanni Paolo II. Dal pianerottolo centrale si dipartono due rampe simmetriche che immettono sulle logge dei piani superiori. Tanto il portico al piano terra che le logge ai piani superiori sono coperte da volte a vela sferiche. Sulle pareti dei pianerottoli intermedi si aprono nicchie destinate a ospitare statue.

II prospetto della scala [10] è segnato da tre ordini di piatte lesene sovrapposte addossate ai pilastri e concluse da semplici capitelli che richiamano quelle adoperate sul prospetto della scala aperta del vicino palazzo Ungaro a Sarno dove le arcate articolate su tre piani sono cinque.

Osservando palazzo Bosco dalla strada, il sistema delle scale a triplici logge sovrapposte emerge imponente rivelandone la presenza quale vero fulcro e cuore dell'intera composizione e quale affaccio privilegiato sul panorama mozzafiato del Vesuvio. 


\section{Conclusioni}

Le vicende dell'architettura civile a Episcopio di Sarno rivelano la necessità di avviare approfondite campagne di studio e di rilevamento degli edifici principali posti lungo l'asse di via Duomo. La morfologia delle soluzioni adottate nelle residenze di Episcopio, il numero e le implicazioni che ne derivano sono tali da richiedere una lettura fatta sulle fonti e sui rilievi qui pubblicati per favorire opportuni approfondimenti sul tema della migrazione del sistema delle scale aperte dai centri principali del regno alle periferie, rimaste spesso ingiustificatamente ai margini degli studi sull'architettura civile tardobarocca e invece meritevoli d'indagini a più ampio spettro.

\section{Bibliografia}

AA. W. (1994), Settecento napoletano: sulle ali dell'aquila imperiale (1707-1734), Electa Napoli, Napoli. ASTARITA, L.; ESPOSITO, A. (ed.) (2003), Itinerary sarnesi: vile e palazzi, Ed. dell'Ippogrifo, Sarno.

BLUNT, A. (2006), Architettura barocca e rococò a Napoli, Londra 1975, Electa, Milano.

CANTONE, G. (2010), La residenza barocca a Napoli e in Campania, in FAGIOLO, M. (ed.), II sistema delle residenze nobiliari: Italia meridionale, De Luca, Roma, pp. 13-41.

- (2003), Campania barocca, Jaka Book, Milano.

CAPOBIANCO M. (1962), «Scale settecentesche a Napoli», L'architettura. Cronache e storia, anno VIII, (8), n. ${ }^{\circ} 84$, pp. $400-417$. DE SETA; DI MAURO; PERONE (1980), Ville Vesuviane. Campania 1, Rusconi, Milano.

GAMBARDELLA, A. (1974), Ferdinando Sanfelice architetto, Arti Grafiche Licenziato, Napoli.

GARMS, J. (2000), Dal viceregno al regno - Napoli, in CURCIO G., KIEVEN E. (a cura di), Storia dell'architettura italiana. // Settecento, 2 vols., Electa, Milano, Vol. I, pp. 260-293.

GRAVAGNuOLO, B. M; ADRIANI, F. (ed.) (2005), Domenico Antonio Vaccaro. Sintesi delle arti, Guida, Napoli.

LABROT, G. (1993), Palazzi napoletani. Storie di nobili e cortigiani 1520-1750, Electa Napoli, Napoli.

- (1979), Baroni in città: residenze e comportamenti dell'aristocrazia napoletana, 1530-1734, Società editrice napoletana, Napoli.

MARÍAS, F. (2005), «Entre Sevilla y Nápoles: Juan Antonio Medrano, Ferdinando Sanfelice y Los Borbones de España de Felipe V a Carlos IIl», Atrio, n. ${ }^{\circ}$ 10/11, pp. 47-56.

NYKJAER, M. (1983), «Architecture and Vision of Space. Some Eighteenth Century Staircases in Southern Italy and their Relationship to Contemporary Concepts of Space", Analecta Romana Instituti Danici, n. ${ }^{\circ} 12$, pp. 149-163.

RUIZ BARBARIN, A. (ed.) (2012), Setenta escalones. La escalera en el tiempo y en el espacio, Fundación Cáser, Madrid.

VÁSQUEZ GESTÁL, P. (2012), «Sólidas, útiles, bellas. Las escaleras y la cultura occidental», in RUIZ BARBARIN, A. (ed.), Setenta escalones. La escalera en el tiempo y en el espacio, Fundación Cáser, Madrid, pp. 14-38.

WARD, A. (1988), The Architecture of Ferdinando Sanfelice, Garland, New-York London. 
